\title{
Introduction to Plasmon Energy Expansion Thermometry
}

B. C. Regan ${ }^{1}$, William A. Hubbard ${ }^{1}$, E. R. White ${ }^{1}$, Rohan Dhall ${ }^{2}$, Stephen B. Cronin ${ }^{2}$, and Shaul Aloni ${ }^{3}$, Matthew Mecklenburg ${ }^{4}$

1. Department of Physics and Astronomy \& California NanoSystems Institute, University of California, Los Angeles, CA, USA

2. Department of Electrical Engineering, University of Southern California, Los Angeles, CA, USA

3. Molecular Foundry, Lawrence Berkeley National Laboratory, Berkeley, CA, USA

4. Center for Electron Microscopy and Microanalysis, University of Southern California, Los Angeles, CA, USA

Obtaining local temperature measurements in a transmission electron microscope (TEM) is challenging. In liquid cell, gas cell, and heating experiments the temperature is typically determined using a thermocouple, optical pyrometer, or calibrated resistor. When using such methods one usually assumes that the temperature in the sample region is spatially homogeneous, since these techniques cannot resolve temperature gradients on small length scales. Here we describe a thermometry technique, plasmon energy expansion thermometry (PEET), which can make local temperature measurements on sub 10-nanometer length scales [1]. The technique does not use an external temperature probe, but rather induces the material under observation to act as a collection of tiny, local thermometers.

In PEET the local density of a material is ascertained from its plasmon energy, and the local temperature is deduced from this density. In a TEM a beam electron can lose energy by creating a charge oscillation (called a plasmon) as it passes through a material. The plasmon's energy is proportional to the square root of the material's valence electron density. Thermal expansion or contraction changes this density as the material is heated or cooled [2]. The temperature-induced plasmon energy shift is thus related to a material's thermal expansion properties.

A plot of this relation is shown in Fig. 1A for aluminum [3]. Typical aluminum electron energy loss spectra (EELS) for two different temperatures are shown in Fig. 1B. The small energy shift, only $\sim-0.5$ $\mathrm{meV} / \mathrm{K}$, is detected by curve fitting both the zero loss peak and the plasmon peak. The plasmon energy is taken to be the energy difference between the peak centers.

Fig. 2 shows a device and the PEET data extracted from it. An electron beam-deposited platinum strap, seen as a dark diagonal line connecting the lower and left contacts, was used to heat one side of the serpentine aluminum wire in the red box (Fig. 2A). Plasmon energy maps of the wire with 0 and 1500 $\mathrm{uW}$ of power delivered to the heater are shown in Figures $2 \mathrm{~B}$ and $2 \mathrm{C}$, respectively. These maps reveal dark, crack-like, structures in the aluminum, which are caused by $\sim 10-20 \mathrm{meV}$ energy shifts at grain boundaries. Combining the two maps according to (C-B)/B (where the letters refer to the panels of Fig. 2) and applying the inverse of the curve in Fig. 1A gives the temperature map Fig. 2D.

The pixel, or spectra acquisition rate was $\sim 76 \mathrm{~Hz}$ for the data of Fig. 2D. The standard deviation of the measured temperature in various small (and thus presumably isothermal) regions ranges from 20 to 30 $\mathrm{K}$, which corresponds to a temperature sensitivity of $\sim 3 \mathrm{~K} / \sqrt{\mathrm{Hz}}$. A variety of spectra acquisition rates yielded this same figure of merit. 
References:

[1] M Mecklenburg et al, Science 347 (2015), p. 629-632.

[2] G Meyer, Zeitschrift fur Physik 148 (1957), p. 61-71.

[3] A J C Wilson, Proceedings of the Physical Society, 54 (1941), p. 235-244.

[4] This work was supported by NSF DMR-1206849, and in part by FAME, one of six centers of STARnet, a Semiconductor Research Corporation program sponsored by MARCO and DARPA. Data presented were acquired at the Center for Electron Microscopy and Microanalysis at the University of Southern California.
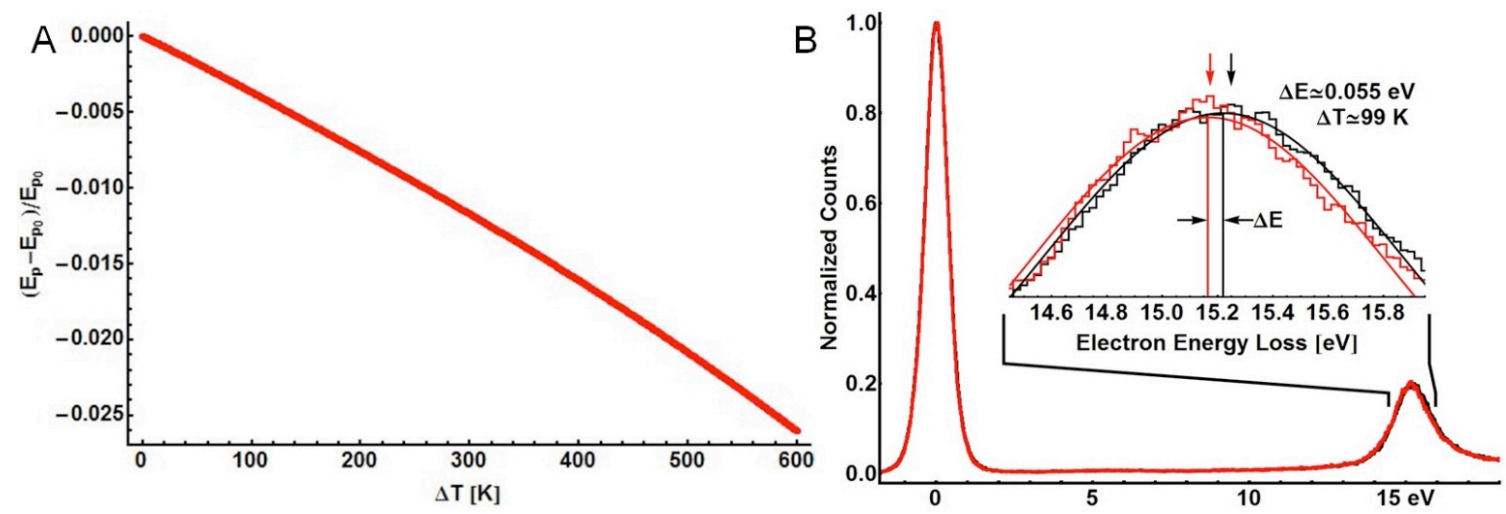

Figure 1. (A) The fractional plasmon energy change vs temperature, calculated from the data in [3]. (B) Two EELS spectra from aluminum, each with a dispersion of $25 \mathrm{meV} / \mathrm{bin}$, at room temperature (black) and $\sim 100 \mathrm{~K}$ hotter (red). The plasmon peak maximum bin for each temperature is indicated with an arrow, and the curve fit centers are indicated with a vertical line.
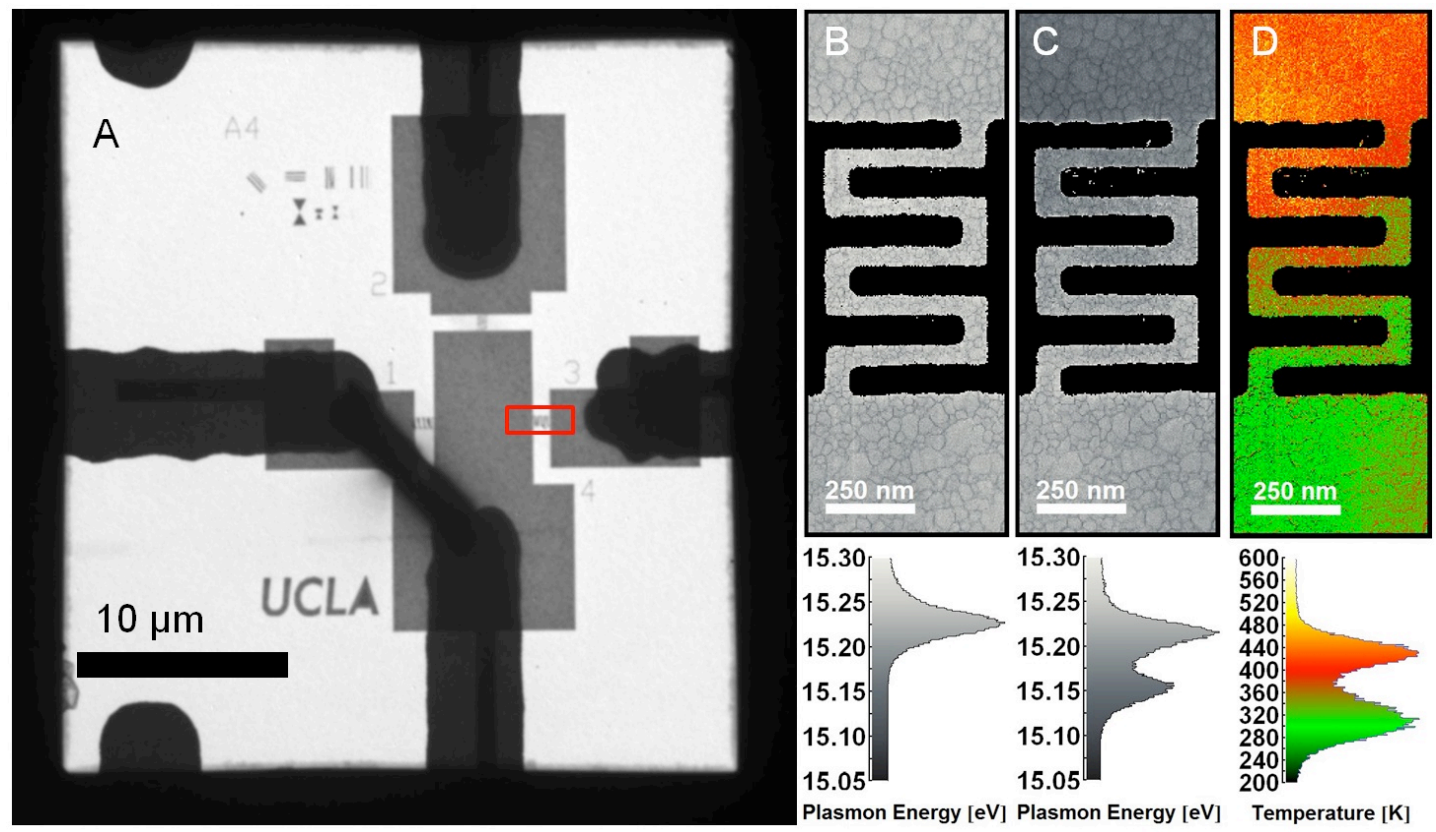

Figure 2. (A) A bright field TEM image of a device. A heater connects the lower and left-hand contacts. PEET was applied to the region indicated with a red rectangle. (B and C) Plasmon energy maps. Each map is $154 \times 398$ pixels, and each pixel is $4 \mathrm{~nm}$ on a side. (D) Temperature map made from images (B) and (C), 151×396 pixels, with the same pixel size as in the plasmon images. A combination intensity scale and pixel count histogram is shown below each image. 